\title{
Measurement of Production Cross Sections for Negative Pions, Kaons, and Protons at 10, 18, and $24 \mathrm{GeV}$
}

$\begin{array}{cc}\text { J. F. Amann } & \text { J. P. DeBrion*** } \\ \text { R. J. Macek } & \text { J. B. Cheze*** } \\ \text { T. W. L. Sanford } & \text { C. Pigot*** } \\ \text { H. A. Thiessen } & \text { E. Pauli*** } \\ \text { O. van Dyck } & \text { D. Simon† } \\ \text { J. Beveridge* } & \text { K. Batzner } \\ \text { A. Burnham* } & \text { C. Steinbach } † \\ \text { G. Ludgate* } & \text { C. E. Roos } \\ \text { F. Massa** } & \text { M. Webster } ¥ \\ \text { G. Marini** } & \text { E. G. H. Williams } \$\end{array}$

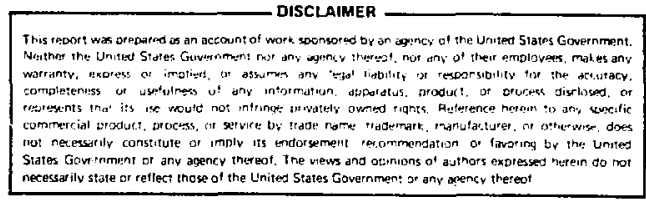

*TRIUMF, University of British Columbia, CANADA V6T 1 W5.

**Universita di Roma, Instituto di Fisica, Piazzale delle Scienze. 5 I-00185 Rome, ITALY.

***Saclay, Centre d'Etudes Nuclaires, B. P. no. 2. F-91190 Gif-sur-Yvette, FRANCE.

tCERIV. CH-1211 Geneva 23. SWITZERLAND.

†Physics Department. Vanderbilt University, Nashville. TN 37203.
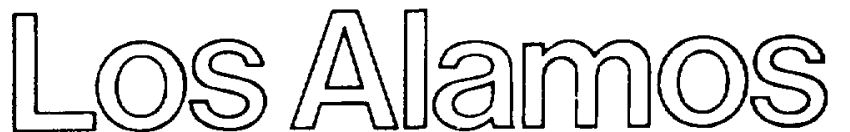




MEASUREMENT OF PRODUCTION CROSS SECTIONS FOR

NEGATIVE PIONS, KAONS, AND PROTONS AT 10, 18, AND $24 \mathrm{GeV}$

by

J. F. Amann, R. J. Macek, T. W. L. Sanford, H. A. Thiessen, O. van Dyck,

J. Beveridge, A. Burnham, G. Ludgate, F. Massa, G. Marini, J. P. DeBrion,

J. B. Cheze, C. Pigot, E. Pauli, D. Simon, K. Batzner, C. Steinbach, C. E. Roos,

M. Webster, and E. G. H. Williams

ABSTRACT

\begin{abstract}
We report here on a measurement of the $0^{\circ}$-production cross sections for low-energy negative secondaries from $10-, 18-$, and $24-\mathrm{GeV}$ protons on a variety of targets. Special emphasis is given to determining the dependence of the cross sections on incident proton energy.
\end{abstract}

\title{
I. INTRODUCTION
}

As the existing meson factories, LAMPF, TRIUMF, and SIN, have reached maturity, there has been a growing interest in the idea of expanding these facilities into so-called "kaon factories." This would involve increasing the primary beam energy to the range of $10-30 \mathrm{GeV}$, but with intensities 100 times greater than existing accelerators such as the Alternating Gradient Synchrotron (AGS) at Brookhaven and the Proton Synchrotron (PS) at CERN. of considerable importance in the planning of such facilities is the dependence of various production cross sections on the primary beam energy. Whereas a number of measurements ${ }^{1-4}$ have existed for many years in this energy region, they have tended to concentrate on higher secondary momenta than would typically be of 
interest at a "kaon factory." In those experiments where measurements were made in the region of interest, there seemed to be substantial disagreements, or the measurements were made under sufficiently different conditions and they could not be directly compared. The intent of this study was to focus on the dependence of the production cross sections with respect to primary beam energy. A secondary goal was to measure absolute cross sections as accurately as possible.

Three primary beam energies were studied $(10,18$, and $24 \mathrm{GeV})$, which encompass the energy region being considered for proposed "kaon factories." Only negatively charged secondaries (pions, kaons, and antiprotons) were studied. A11 three secondaries were measured at momenta of 1.06 and $1.40 \mathrm{GeV} / \mathrm{c}$ with only pions being seen at $0.40 \mathrm{GeV} / \mathrm{c}$.

\section{EXPERTMENTAL PROCEDURE}

The production measurements were carried out in the $\mathrm{k} 24$ beam line at the CERN PS, using the data acquisition system of the Rome-Saclay-Vanderbilt group. A complete description of this apparatus may be found elsewhere. ${ }^{5}$ The beam line is shown in Fig. 1. Protons from the PS impinged on a variety of thin production targets. The resulting secondaries were then steered by the bending magnets BM1 and BM2 and the vertical bending magnets VM1 and VM2 on to the experimental detectors. Only these beam elements were required for the measurements; the separators, all quadrupoles, and the VENUS spectrometer magnet were turned off. At each of the secondary momenta studied, the central momentum of the channel was fixed by the first bending magnet BM1. The remaining elements BM2, VM1, and VM2 would then be scanned for maximum coincidence counting rate. Normally the two vertical bending magnets would be moved together as a pair, maintaining a fixed ratio between their current settings. The effect of altering this ratio was checked and found to be negligible. The momentum and mass slits ( $\mathrm{C} 3$ and $\mathrm{C} 4$ respectively) and the first vertical solid-angle slit (Cl) were opened sufficiently wide to have no effect on the beam flux; control of the overall particle flux was achieved by varying the primary beam current and the horizontal acceptance collimator C2. A11 collimators were positioned at the beginning of the run and left unchanged throughout all subsequent measurements. 


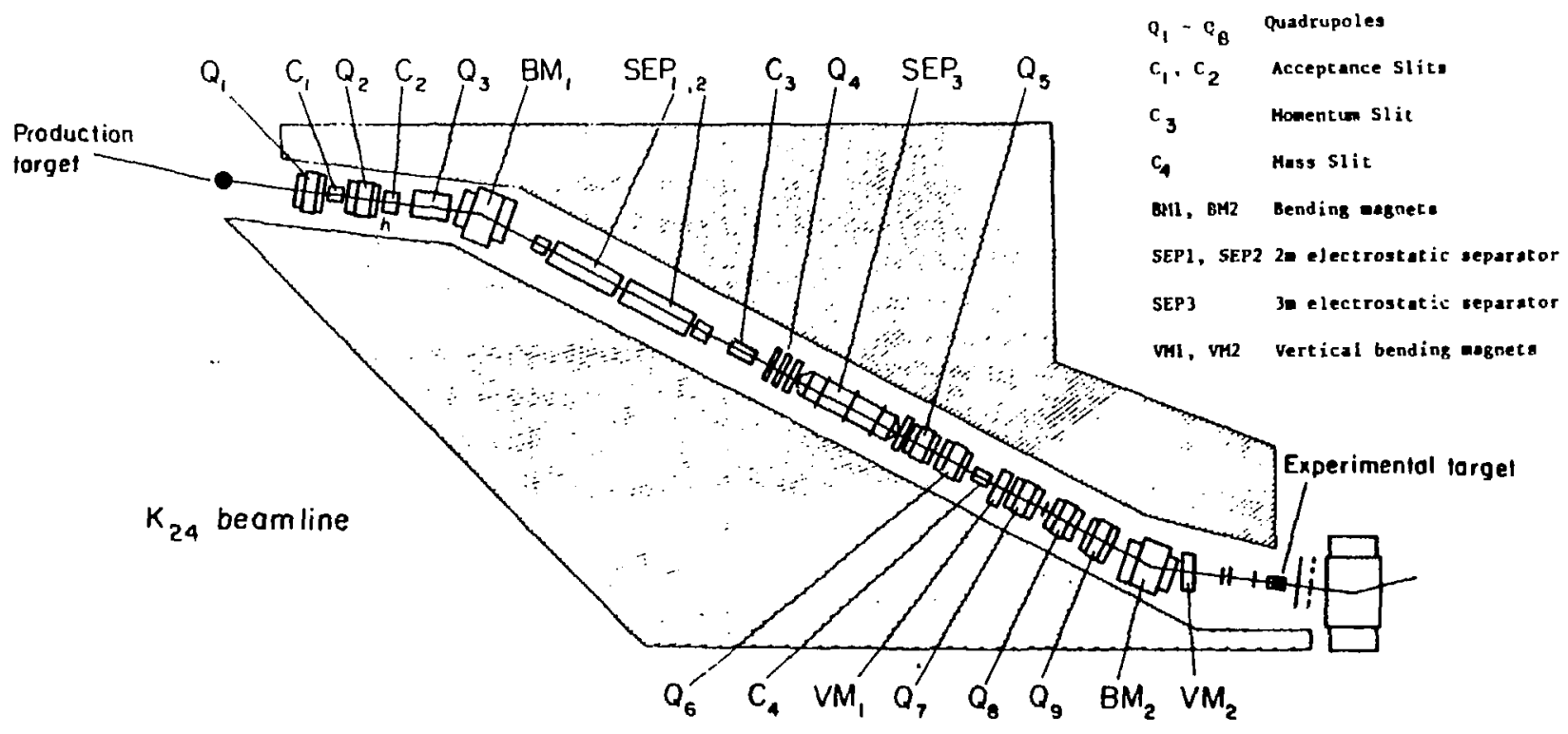

Fig. 1. Layout of K24 beam 1ine. 
Figure 2 shows the experimental layout. Two PILOT-U scintillation counters Sl and S2 provided the coarse beam definition. The aerogel Cêrenkov counter C0, situated immediately downstream of $\mathrm{S} 2$, was used as a veto counter for half the runs to reduce the pion and electron comporient in the trigger. The efficiency of this counter for vetoing pions was typically $98-99 \%$. Although not directly used for the yield measurements, a second aerogel counter Cl was used to determine the undesired efficiency of $\mathrm{CO}$ in vetoing kaons and antiprotons. A more complete discussion of the construction and operating characteristics of the counters has been published previous $1 y, 6,7$

The gas Cêrenkov C4 contained Freon at a pressure of 1 atm. Analysis of its pulse height spectrum allowed a separation of the pi/mu fraction from the electron component. In addition, the counts of kaons and antiprotons in this counter could be used to estimate the fraction of pions misidentified as electrons (because of random coincidences between singles in $\mathrm{C} 4$ and an event).

The primary beam current was determined by the standard secondary-emission monitor (SEC) located upstream of the production target. Only a fraction of the slow extracted beam, typically 5 $\cdot 10^{11}$ protons/pulse, was directed at our production targets. The SEC was calibrated using a toroid current monitor at current levels comparable to those used in actual data taking. Position of the beam on the production targets was monitored periodically by inserting a phosphor viewed by a TV camera in place of the customary target. Beam positions appeared to remain stable to within $\pm 2 \mathrm{~mm}$ throughout the measurements.

\section{DATA ANALYSIS}

Identification of the various beam components was made by a combination of a measurement of the time of flight (TOF) of the particles between $S 1$ and $S 2$ and their pulse height in the gas Cêrenkov counter $\mathrm{C} 4$.

The basic beam trigger was formed from the coincidence

$$
\mathrm{BEAM}=\mathrm{S} 1 \cdot \mathrm{S} 2 \cdot \overline{\mathrm{AS} 2},
$$

where S1 - S2 gives the basic beam definition as mentioned earlier, and AS2 vetos particles in the periphery of the beam that would not intersect the central region of the Cêrenkov counters. This trigger was sufficient to measure the pion and electron yields solely on the basis of their pulse height in $\mathrm{C} 4$. 


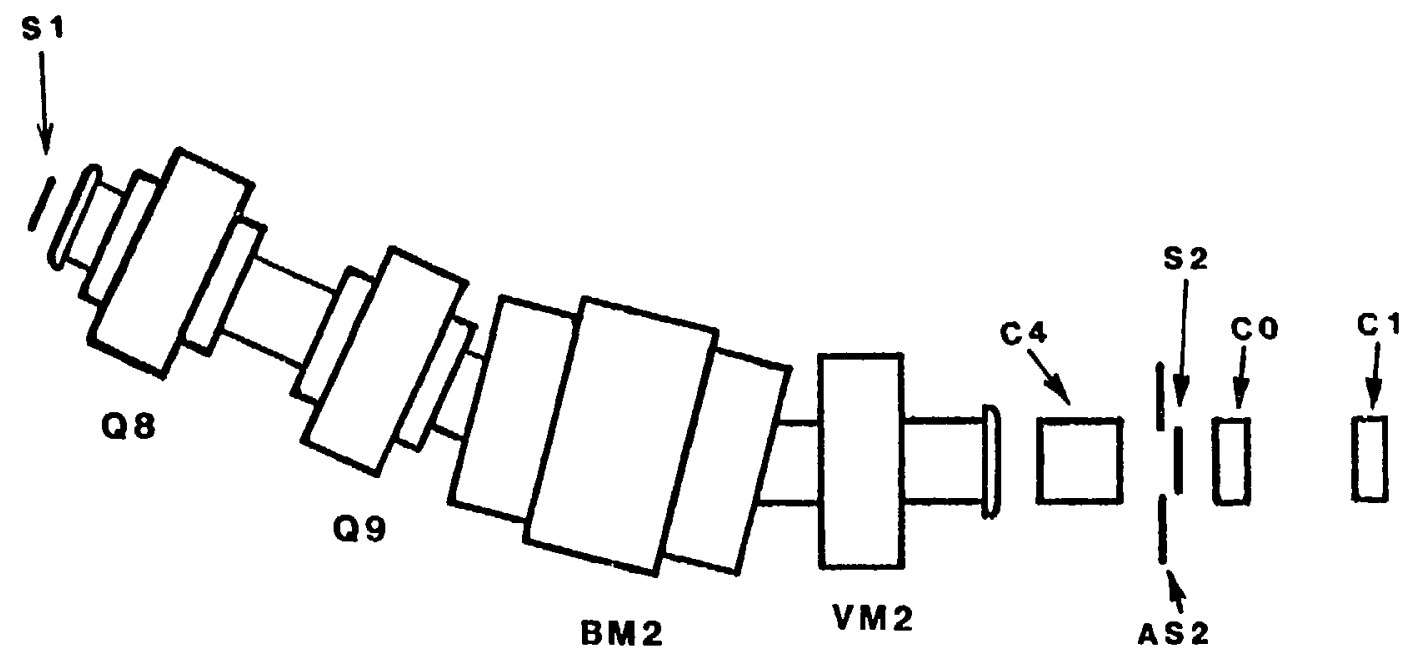

Fig. 2. Experimental detector arrangement. 
To facilitate determination of the kaon and antiproton yields, the $c 0$ counter was used in anticoincidence to suppress the $\mathrm{pi} / \mathrm{mu} / \mathrm{e}$ content of the beam. The trigger requirement thus became

$$
\mathrm{KAON} \mathrm{BEAM}=\mathrm{S} 1 \cdot \mathrm{S} 2 \cdot \overline{\mathrm{AS} 2} \cdot \overline{\mathrm{CO}} \cdot
$$

In this case the fraction of all KAON BEAM events that were kaons and antiprotons was determined solely from the TOF spectrum.

\section{A. Corrections to the Data}

A number of corrections have been applied to the basic yields as determined above. The first involves the subtraction of the yield measured without a production target in place. Such a measurement was made for each combination of primary beam energy and secondary channel momentum. The yields found with no target in place ranged from $5-20 \%$ of target-in yields depending on target. The most likely source of these events is the SEC located upstream of the production target. These events could be seen with some probability because of the $0^{\circ}$ angle at which the $\mathrm{K} 24$ beam line views the production target.

A pair of corrections involves the gas Cêrenkov counter $\mathrm{C} 4$. The first of these corrects for the fraction of true pion events, which resulted in an above threshold signal in the $\mathrm{C} 4$ pulse height spectrum. These erroneous signals were due to the random coincidence of an electron with another particle within the relatively broad time gate on the $\mathrm{ADC}$. We were able to estimate the fraction of such misidentified events by utilizing the fact that beam parameters remained relatively constant between pairs of runs with $\mathrm{CO}$ in and out of the veto. Although at secondary momenta of 1.06 and $1.40 \mathrm{GeV} / \mathrm{c}$, the TOF spectrum cannot be used to separate pions and electrons, we were able to examine what fraction of events with kaon- and antiproton-like values for the TOF also have above-threshold values in $\mathrm{C} 4$, This fraction ranges from $2-10 \%$ and appears to track well with beam flux, thus supporting the hypothesis that these anomalous events were caused by random coincidences. The validity of extrapolating this number to pions is further supported by the fact that the fraction of misidentified kaons and misidentified antiprotons was always the same within statistics. 
The second correction to the raw data involving $\mathrm{C} 4$ was due to its inefficiency for identifying electrons. The actual efficiency was measured during the runs taken at a channel momentum of $0.40 \mathrm{GeV} / \mathrm{c}$. At this momentum the TOF spectrum was capable of separating pions, muons, and electrons. Thus, for events identified as electrons by TOF, it was determined that the average efficiency of $\mathrm{C} 4$ was $98 \%$.

Another correction to the raw data involves the possibility that while CO was used as a veto, it falsely vetoed some kaons and antiprotons. There are several possible causes. The first is the random coincidence of an electron or pion with a kaon or antiproton within the coincidence window of the veto circuitry. These random coincidences were not measured directly, but the fraction of such events can be estimated from the random coincidences involving C4 discussed above, the relative gate widths for the $C 0$ veto ( $20 \mathrm{~ns}$ ), and the C4 ADC (100 ns). On this basis, between 0.5 and $2 \%$ of kaons and antiprotons were vetoed because of random coincidences with an electron or pion.

A second category of false vetos arises from the production of a sufficiently fast electron or pion by the kaon or antiproton either within co or just upstream of it. Production of sufficiently energetic delta rays (1.13-MeV electrons) to exceed the Cêrenkov threshold in $\mathrm{C} 0$ is very improbable $(<1 \%)$ even for the case of $1.4-\mathrm{GeV} / \mathrm{c}$ kaons. Production of fast pions through absorption of the kaon or antiproton is also a small effect $(<1.5 \%)$ based on the thickness of CO ( 0.015 absorption lengths), even if every interacting kaon or antiproton produced a sufficiently fast secondary.

A final possibility for false vetos is that at $1.4 \mathrm{GeV} / \mathrm{c}$ kaons are not very far below threshold. Hence, statistical fluctuations in the number of photoelectrons produced would give rise to some triggering of $\mathrm{C} 0$.

In order to quantify this last class of false vetos, a series of measurements were made at a secondary momentum of $1.4 \mathrm{GeV} / \mathrm{c}$. In this case the counter $\mathrm{Cl}$ was installed as a veto in place of $\mathrm{CO}$ to enhance the kaon and pion component of the trigger. Yields were then measured, both with and without $\mathrm{CO}$ being used as an additional veto. Comparison of the yields in both cases indicated that $2.5 \pm 7.1 \%$ of the antiprotons and $8.3 \pm 13.5 \%$ of the kaons were falsely vetoed by $\mathrm{C} 0$. These corrections have been applied to kaon and antiproton cross sections at both 1.06 and $1.40 \mathrm{GeV} / \mathrm{c}$. It should be mentioned that this form of calibration should adequately measure the third category of false vetos but would not measure those caused by random coincidences nor those 
caused by delta rays or interactions producing fast pions due to the close proximity of $\mathrm{CO}$ and $\mathrm{Cl}$.

A final correction involves multiple scattering of the particles in $\mathrm{S} 1$ and the accompanying vacuum window and intervening air space. The transport geometry of the beam line is such that both $\mathrm{S} 1$ and $\mathrm{S} 2$ define nearly the same region of theta-delta phase space. Multiple scattering in the vicinity of $S 1$ may cause some particles, which otherwise would have passed through $\mathrm{S} 2$, to fail to do so. A Monte Carlo simulation of this effect has been performed and the resulting multiplicative correction factors are listed in Table $I$.

After all above corrections had been applied to the basic yields, a correction was made for the decay in flight of pions and kaons in the $36.0-\mathrm{m}$ length from the production target to $\mathrm{S} 2$. It was assumed that no products from such decays remained within the acceptance phase space of the detection system. The applicable survival fractions are given in Table II. The absolute cross sections, including all corrections described above, are listed in Tables III - V.

Because the electron fraction of the beam is so strongly a function of target thickness, we do not calculate cross sections for electrons. Rather, we have simply listed in Table VI what fraction of the beam consisted of electrons. For the $0.40-\mathrm{GeV} / \mathrm{c}$ data this was determined directly by TOF. At the higher momenta, $\mathrm{C} 4$ was used as described above.

\section{B. Error Analysis}

Other than the statistical errors involved in estimating the number of events of each type after background subtraction, a number of possible systematic errors remain.

The first of these is nonstatistical fluciuations in the the number of BEAM events per SEC count. We have estimated this uncertainty to be $\pm 5.6 \%$ by comparing the ratio BEAM/SEC for all pairs of runs taken with $C 0$ in and out as a veto. This is generally consistent with the variations found when the primary beam current was varied by factors of 2 and when the beam was moved $\pm 2 \operatorname{mm}$ on the production target. This error affects all cross sections equally and has been incorporated into the errors listed in Tables III - V.

The following errors affect only the ratios of cross sections between different particle types or between the same particle types at different 
secondary momenta, but are not dependent on the primary beam energy. They have not been included in the error estimates listed in Tables III - V.

The first of these results from an uncertainty in the $P_{0}$, the central momentum of the channe1. This results in an uncertainty in the decay correction factors that must be applied for pions and kaons. We have estimated the uncertainty in $P_{0}$ at $\pm 1 \%$ resulting in uncertainties in the absolute cross section of $\pm 4 \%(2.5 \%)$ for kaons at $1.06(1.40) \mathrm{GeV} / \mathrm{c}$ respectively, and less than $1 \%$ for pions at either momentum.

Another uncertainty involving decay corrections for pions and kaons is due to possible error in the decay length assumed for the channel. Assuming an uncertainty of $\pm 0.5 \mathrm{~m}$ about the nominal $36.0-\mathrm{m}$ length from production target to S2 results in $\pm 6.0(4.5) \%$ error in the kaon cross sections at $1.16(1.40) \mathrm{GeV} / \mathrm{c}$. Again the uncertainty for pions is less than $1 \%$.

Although the measured efficiency of $\mathrm{CO}$ for vetoing good kaons and antiprotons was small, 8.3 and $2.5 \%$ respectively, we have estimated an added uncertainty in the absolute cross section of $\pm 5 \%$ in each case to account for possible vetos caused by other unmeasured mechanisms as discussed above.

An uncertainty in the scale of the absolute cross sections is due to difficulties in estimating the solid angle of the channel. Varying the nature of the assumed fringe field of the dipoles within reasonable limits results in a $\pm 10 \%$ variation in the effective solid angle. This affects all particles equally.

Adding all these effects in quadrature implies that the additional systematic uncertainties listed in Table VII apply to the errors quoted for the absolute cross sections in Tables III - V.

\section{DISCUSSION}

The most striking feature of the present data is the rapid rise of the cross sections with incident proton energy. Yields for all particles rise faster than the increase in beam energy, with the most rapid increase being for the production of antiprotons. This is illustrated for the carbon target in Fig. 3. All other targets show a similar energy dependence. The dependence of the cross sections on the secondary particle momenta is shown for the carbon target in Figs. 4-6 for proton energies of 10,18 , and $24 \mathrm{GeV}$ respectively. Also shown are predictions for the $0^{\circ}$ cross sections on carbon made using the Sanford-Wang parameterization, ${ }^{8}$ including kinematic reflection. Figure 3 also 


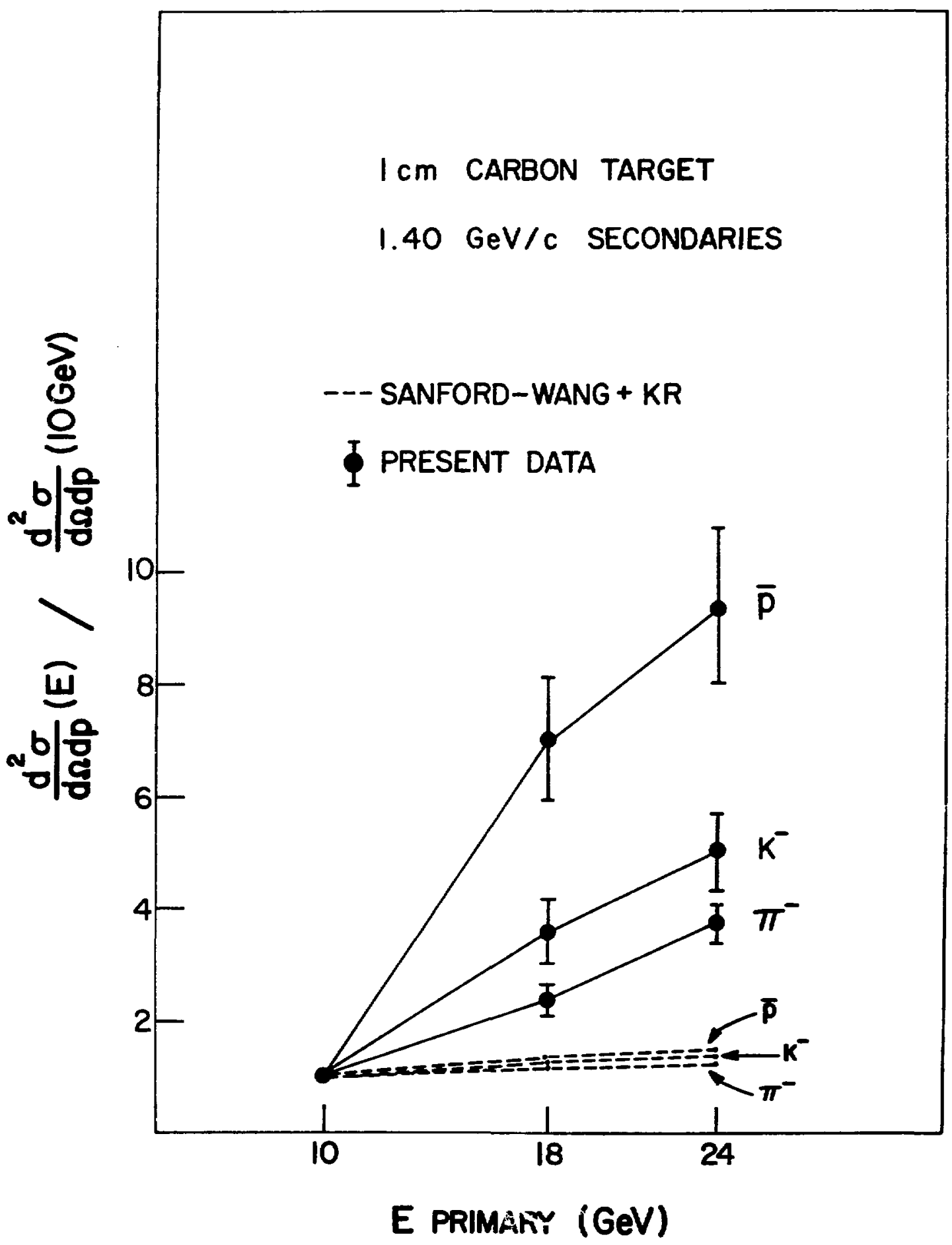

Fig. 3. Energy dependence of production cross sections on 1$\mathrm{cm}$ carbon target normalized to $10 \mathrm{GeV}$ cross section. 


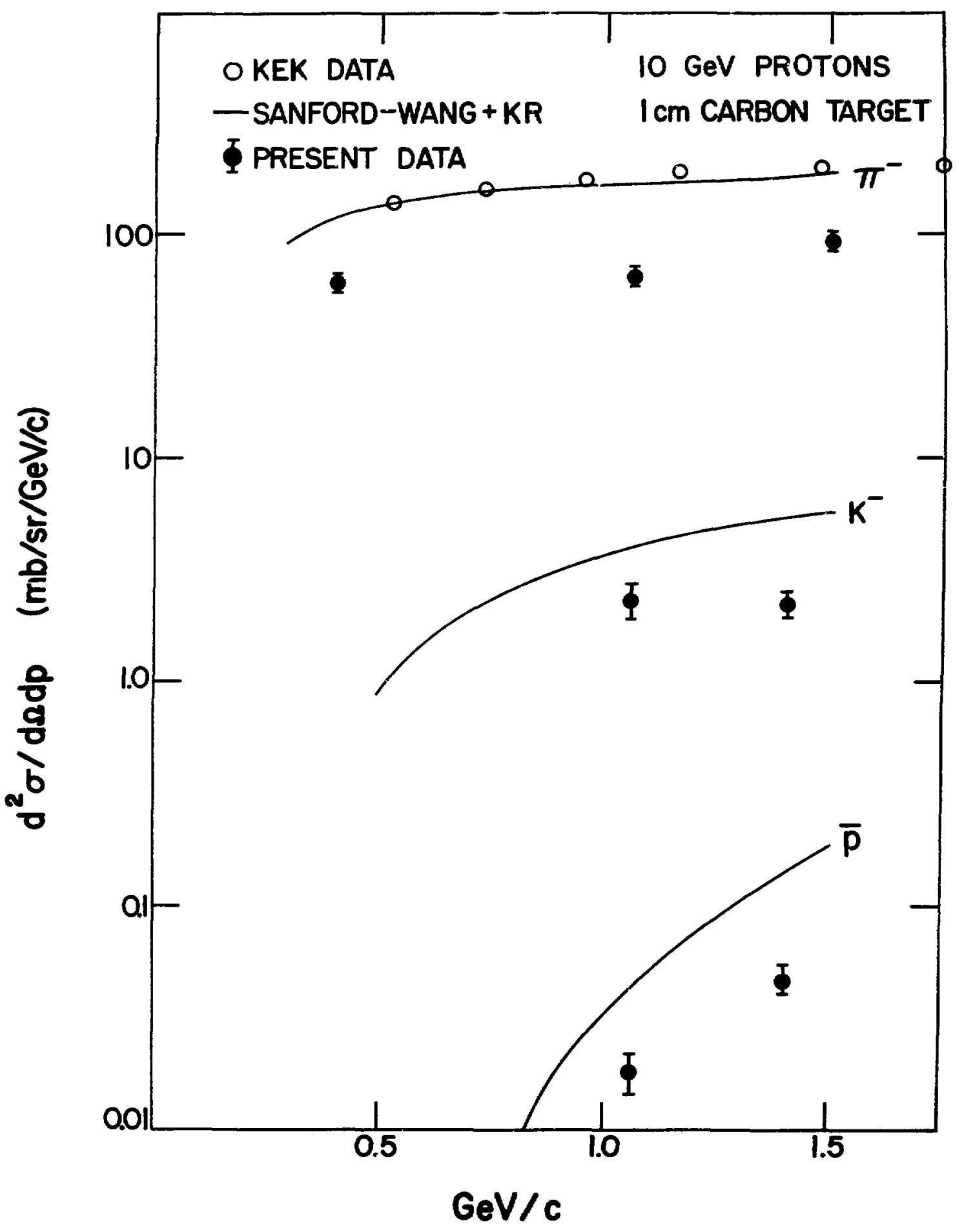

Fig. 4. 10-GeV production cross sections on 1-cm carbon target. 




Fig. 5. 18-GeV production cross sections on 1-cm carbon target. 




Fig. 6. 24-GeV production cross sections on 1-cm carbon target. 
shows the recent measurements of Yamamoto et a1. ${ }^{9}$ at KEK for $12-\mathrm{GeV}$ protons on a beryllium target.

It is clear from these plots that the Sanford-Wang parameterization does an adequate job or predicting the relative yields between different types of secondaries, as well as the shape of the cross section wich respect to secondary momentum. However, it does very poorly at predicting the dependence on incident proton energy. Indeed, Sanford-Wang predicts very little increase of the $0^{\circ}$ cross sections with energy, whereas we measure substantial increases for all secondaries.

The disagreement between our measurements and those of $\operatorname{Ref} 9$ is not understood. One possibility is that there is sharp angular dependence of the cross sections at small angles. Our measurements were performed with a rather small angular acceptance $\left( \pm 0.5^{\circ}\right)$ as compared with that of Ref. $9\left( \pm 2.9^{\circ}\right)$. While Sanford-Wang predicts just such an increase for pions at small angles, it predicts that antiproton cross sections will decrease. By way of confirmation of our results, a series of measurements were performed on the K26 beam line at CERN concurrent with those reported here. These measurements concentrated only on the low-momentum secondaries $(400 \mathrm{MeV} / \mathrm{c})$. By the use of a much shorter channel and an electrostatic separator, it was possible to measure cross sections for kaons and antiprotons not obtainable at $k 24$. The resulting cross sections ${ }^{10}$ are listed in Appendix $A$ and are in good agreement with our pion measurements at both $10 \mathrm{GeV}$ and $24 \mathrm{GeV}$.

\section{ACKNOWLEDGMENTS}

We wish to thank Lothar Hoffman for his interest and enthusiasm for this experiment, as well as Tjeerd Ketel ard the other members of the Heidelberg/Saclay/Strasbourg collaboration who made the measurements on the K26 beam line. H. A. Thiessen would especially like to thank Dr. B. Povh and the Max Planck Institute for their hospitality during the year in which these measurements were planned and carried out. 
1. D. Dekkers, J. A. Geibe1, R. Mermod, G. Weber, T. R. Willitts, K. Winter, B. Jordan, M. Vivargent, N. M. King, E. J. N. Wilson, Fhys. Rev. 137, 1962 (1965).

2. R. A. Lundy, T. B. Novey, D. D. Yavanovitch, and V. L. Telegdi, Phys. Rev. Lett. 14, 504 (1966).

3. W. F. Baker, R. L. Cool, W. E. Jenkins, T. F. Kycia, S. J. Lindenbaum W. A. Love, D. Luers, J. A. Niederer, S. Ozaki, A. L. Read, J. J. Russe11, L. C. L. Yuan, Phys. Rev. Lett. 7, 101 (1961).

4. V. L. Fitch, S. L. Meyer, and P. A. Pirone, Phys. Rev. 126, 1849 (1962).

5. G. D'Agostini, M. Arrignon, G. Auriemma, A. Bezaguet, J. P. DeBrion, A. Caillet, J. B. Cheze, J. Derre, G. Ducastaing, G. Durand, E. Gennari, M. Lemoine, R. Llosa, G. Mare1, G. Marini, G. Martellotti, F. Massa, A. Nigro, E. Pauli, C. Pigot, A. Rambaldi, S. Reucroft, C. E. Roos, B. Rothan, K. Schultze, A. Sciubba, S. Tentindo, G. Vrana, M. Webster, and E. G. H. Williams, CERN -EP/81-17, (1981).

6. G. D'Agostini, G. Marini, G. Martellotti, F. Massa, and A. Sciubbe, CERN-EP/80-228 (1980).

7. J. P. DeBrjon, A. Caillet, J. B. Cheze, J. Derre, G. Mare1, E. Pauli, C. Pigot, and G. Vrana, Nuc1. Instrum. Methods 179, 61 (1981).

8. J. R. Sanford and C. L. Wang, BNL-112;; and 11479 (1967).

9. A. Yamamoto, KEK 81-13 (1981).

10. T. Kete1, Nucleus Heidelberg -- Interna1 Note (1981). 
TABLE I

CORRECTION FACTORS FOR MULTIPLE SCATTERING

$\begin{array}{lrrr}\mathrm{P}(\mathrm{Gev} / \mathrm{c}) & \underline{\mathrm{\pi}^{-}} & \underline{\mathrm{K}^{-}} & \underline{\overline{\mathrm{P}}} \\ 0.400 & 1.62 & -\cdots & -- \\ 1.050 & 1.12 & 1.15 & 1.21 \\ 1.400 & 1.05 & 1.06 & 1.10\end{array}$

TABLE II

SURVIVAL FRACTIONS OF PIONS ANI) KAONS

\begin{tabular}{llll}
$P(\mathrm{GeV} / \mathrm{C})$ & $\mathrm{f}_{\mathrm{S}}\left(\pi^{-}\right)$ & $\mathrm{f}_{\mathrm{S}}\left(\mathrm{K}^{-}\right)$ \\
\cline { 2 - 2 } 0.400 & 0.200 & & - \\
1.060 & 0.545 & & 0.0109 \\
1.400 & 0.631 & & 0.0326
\end{tabular}


TABLE III

PRODUCTION CROSS SECTIONS AT $10 \mathrm{GeV}$

$$
\mathrm{d}^{2} \sigma / \mathrm{d} \Omega \mathrm{dP} \quad(\mathrm{mb} / \mathrm{sr} / \mathrm{GeV} / \mathrm{c})
$$

Channe1 Momentum $=0.40 \mathrm{GeV} / \mathrm{c}$

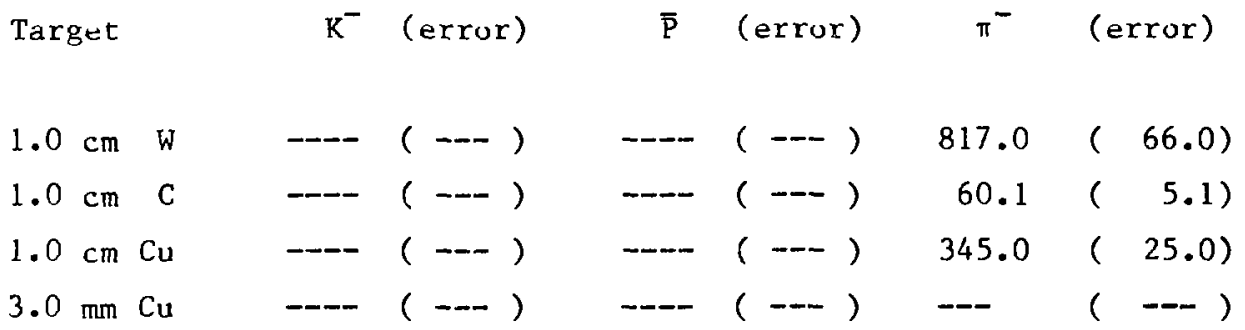

Channe1 Momentum $=1.06 \mathrm{GeV} / \mathrm{c}$

Target $\mathrm{K}^{-}$(error) $\mathrm{P}$ (error) $\pi^{-}$(error)

$\begin{array}{lllllllll}1.0 \mathrm{~cm} & W & 17.6 & (1.7) & 0.0803 & (0.0114) & 543.0 & (36.0) \\ 1.0 \mathrm{~cm} \mathrm{C} & 2.34 & (0.41) & 0.0182 & (0.0037) & 64.8 & ( & 5.1) \\ 1.0 \mathrm{~cm} \mathrm{Cu} & 7.72 & (0.89) & 0.0592 & (0.0077) & 260.0 & (17.0) \\ 3.0 \mathrm{~mm} \mathrm{Cu} & --- & (--) & -- & (-) & -\infty & (--)\end{array}$

Channel Momentum $=1.40 \mathrm{GeV} / \mathrm{c}$

$\begin{array}{llllll}\text { Target } & \mathrm{K}^{-} & \text {(error) } & \overline{\mathrm{P}} & \text { (error) } \pi^{-} & \text {(error) }\end{array}$

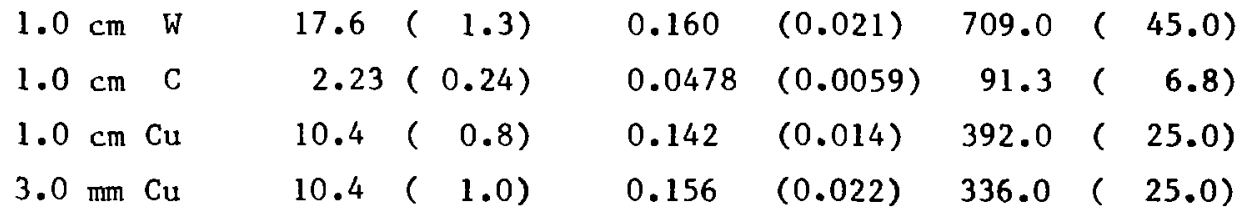


PRODUCTION CROSS SECTIONS AT $18 \mathrm{GeV}$

$$
\mathrm{d}^{2} \sigma / \mathrm{d} \Omega \mathrm{dP} \quad(\mathrm{mb} / \mathrm{sr} / \mathrm{GeV} / \mathrm{c})
$$

Channe1 Momentum $=0.40 \mathrm{GeV} / \mathrm{c}$

\begin{tabular}{|c|c|c|c|c|c|}
\hline Target & $k^{-}$ & (error) & $\bar{P}$ & (error) & $\pi^{-} \quad($ error $)$ \\
\hline $1.0 \mathrm{~cm} \mathrm{~W}$ & --- & $(--)$ & --- & $(--)$ & $1365.0(131.0)$ \\
\hline $1.0 \mathrm{~cm} \mathrm{C}$ & -- & $(--)$ & --- & $(--)$ & $127.0(11.0)$ \\
\hline $1.0 \mathrm{~cm} \mathrm{Cu}$ & -- & $(--)$ & $-\cdots$ & $(--)$ & $594.0(55.0)$ \\
\hline $.0 \mathrm{~mm}$ & --- & $(--)$ & -- & $(--)$ & $(--$ \\
\hline
\end{tabular}

Channel Momentum $=1.06 \mathrm{GeV} / \mathrm{c}$

Target $\mathrm{K}^{-}$(error) $\overline{\mathrm{P}}$ (error) $\pi^{-}$(error)

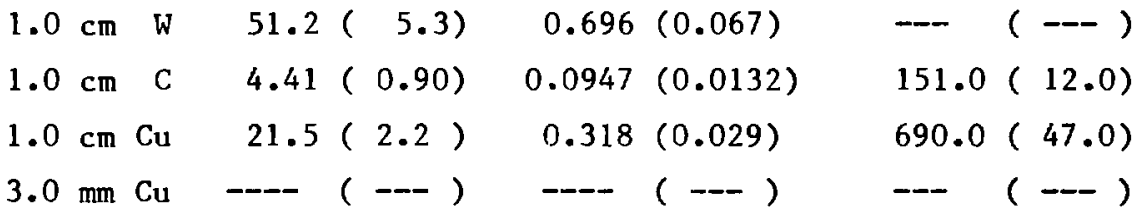

Channe1 Momentum $=1.40 \mathrm{GeV} / \mathrm{c}$

$\begin{array}{lcccc}\text { Target } & \mathrm{K}^{-} \text {(error) } & \overline{\mathrm{P}} \text { (error) } & \pi^{-} \text {(error) } \\ 1.0 \mathrm{~cm} \mathrm{~W} & 45.1(3.5) & 1.38(0.11) & 1453.0(96.0) \\ 1.0 \mathrm{~cm} \mathrm{C} & 7.70(0.93) & 0.335(0.037) & 200.0(15.0) \\ 1.0 \mathrm{~cm} \mathrm{Cu} & 23.0(1.9) & 0.933(0.072) & 632.0(41.0) \\ 3.0 \mathrm{~mm} \mathrm{Cu} & ---(---) & ---(--) & -\cdots(--)\end{array}$


PRODUCTION CROSS SECTIONS AT $24 \mathrm{GeV}$

$$
\mathrm{d}^{2} \sigma / \mathrm{d} \Omega \mathrm{dP} \quad(\mathrm{mb} / \mathrm{sr} / \mathrm{GeV} / \mathrm{c})
$$

Channel Momentum $=0.40 \mathrm{GeV} / \mathrm{c}$

\begin{tabular}{|c|c|c|c|c|c|}
\hline Target & $\mathrm{K}^{-}$ & (error) & $\overline{\mathbf{P}}$ & (error) & $\pi^{-}$(error) \\
\hline $1.0 \mathrm{~cm} \mathrm{~W}$ & --- & $(--)$ & - & $(-\infty)$ & $1840.0(199.0)$ \\
\hline $1.0 \mathrm{~cm} \mathrm{C}$ & ---- & $(--)$ & - & $(--)$ & $173.0(16.0)$ \\
\hline $0 \mathrm{~cm} \mathrm{Cu}$ & --- & $(--)$ & ---- & $(--)$ & $869.0(71.0)$ \\
\hline $.0 \mathrm{~nm}$ & --- & $(--)$ & --- & $(---)$ & $707.0(76.0)$ \\
\hline
\end{tabular}

Channe1 Momentum $=1.06 \mathrm{GeV} / \mathrm{c}$

$\begin{array}{lccrrr}\text { Target } & \mathrm{K}^{-} \text {(error) } & \mathrm{P} \text { (error) } & \pi^{-} \text {(error) } \\ & & & & & \\ .0 \mathrm{~cm} \quad \mathrm{~W} & 63.5 & (6.1) & 0.797(0.073) & 1839.0(123.0) \\ .0 \mathrm{~cm} \mathrm{C} & 6.36(0.79) & 0.149(0.014) & 264.0(19.0) \\ .0 \mathrm{~cm} \mathrm{Cu} & 28.7(2.4) & 0.504(0.038) & 928.0(62.0) \\ 3.0 \mathrm{~mm} \mathrm{Cu} & 29.1(2.8) & 0.540(0.046) & 933.0(66.0)\end{array}$

Channe1 Momentum $=1.40 \mathrm{GeV} / \mathrm{c}$

$\begin{array}{lllll}\text { Target } & \mathrm{K}^{-} \text {(error) } & \overline{\mathrm{P}} \text { (error) } & \pi^{-} \text {(error) } \\ 1.0 \mathrm{~cm} \mathrm{~W} & 76.0(5.7) & 2.35(0.18) & 2667.0(168.0) \\ 1.0 \mathrm{~cm} \mathrm{C} & 11.0(1.0) & 0.450(0.040) & 335.0(24.0) \\ 1.0 \mathrm{~cm} \mathrm{Cu} & 40.2(3.0) & 1.33(0.10) & 1364.0(85.0) \\ 3.0 \mathrm{~mm} \mathrm{Cu} & 42.8(3.7) & 1.64(0.14) & 1232.0(87.0)\end{array}$


TABLE VI

ELECTRON FRACTION OF BEAM AT S2

\begin{tabular}{|c|c|c|c|}
\hline \multicolumn{4}{|c|}{ Proton energy } \\
\hline \multirow[t]{2}{*}{ Target } & \multicolumn{2}{|c|}{ Momentum } & $(\mathrm{GeV} / \mathrm{c})$ \\
\hline & 0.40 & 1.06 & 1.40 \\
\hline $1.0 \mathrm{~cm} \mathrm{~W}$ & 0.76 & 0.43 & 0.25 \\
\hline $1.0 \mathrm{~cm} \mathrm{C}$ & 0.36 & 0.13 & 0.07 \\
\hline $1.0 \mathrm{~cm} \mathrm{Cu}$ & 0.69 & 0.39 & 0.24 \\
\hline $3.0 \mathrm{~mm} \mathrm{Cu}$ & ---- & ---- & 0.14 \\
\hline Proton & energy & $18 \mathrm{Ge}$ & \\
\hline \multirow[t]{2}{*}{ Target } & \multicolumn{2}{|c|}{ Momentum } & $(\mathrm{GeV} / \mathrm{c})$ \\
\hline & 0.40 & 1.06 & 1.40 \\
\hline $1.0 \mathrm{~cm} \mathrm{~W}$ & 0.88 & --- & 0.41 \\
\hline $1.0 \mathrm{~cm} \mathrm{C}$ & 0.63 & 0.23 & 0.12 \\
\hline $1.0 \mathrm{~cm} \mathrm{Cu}$ & 0.82 & 0.54 & 0.36 \\
\hline $3.0 \mathrm{~mm} \mathrm{Cu}$ & $-\cdots$ & -- & --- \\
\hline
\end{tabular}

Proton energy $=24 \mathrm{GeV}$

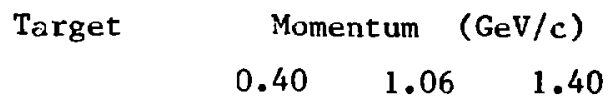

$\begin{array}{lllll}1.0 \mathrm{~cm} & W & 0.83 & 0.76 & 0.53\end{array}$

$\begin{array}{lllll}1.0 \mathrm{~cm} & \mathrm{C} & 0.72 & 0.26 & 0.15\end{array}$

$\begin{array}{llll}1.0 \mathrm{~cm} \mathrm{Cu} & 0.87 & 0.66 & 0.44\end{array}$

$\begin{array}{llll}3.0 \mathrm{~mm} \mathrm{Cu} & 0.81 & 0.44 & 0.28\end{array}$ 
TABLE VII

SYSTEMATIC UNCERTAINTIES IN ABSOLUTE CROSS SECTIONS

$\begin{array}{cccc}\mathrm{P}(\mathrm{GeV} / \mathrm{c}) & \pi^{-} & \mathrm{K}^{-} & \mathbf{P} \\ 0.400 & \pm 11 \% & --- & --- \\ 1.060 & \pm 10 \% & \pm 13 \% & \pm 11 \% \\ 1.400 & \pm 10 \% & \pm 12 \% & \pm 11 \%\end{array}$


APPENDIX A

The following are cross sections measured for $400-\mathrm{MeV} / \mathrm{c}$ negatively charged secondaries from a 3-mm copper production target at 10- and 24-GeV primary proton energy. Measurements were made on the K26 beam line at the CERN PS and in the case of $\pi^{-}$and $\mathrm{K}^{-}$, are corrected for decay in the 11.6-m distance from production target to final detector.

\begin{tabular}{ccc} 
Particle & $\begin{array}{l}\text { i0-GeV } \\
\text { Cross Section } \\
\mathrm{mb} /(\mathrm{sr} / \mathrm{GeV} / \mathrm{c})\end{array}$ & $\begin{array}{l}\text { 24-GeV } \\
\text { Cross Section } \\
\mathrm{mb} /(\mathrm{sr} / \mathrm{GeV} / \mathrm{c})\end{array}$ \\
\cline { 2 - 2 } & 300.0 & 680.0 \\
$\pi^{-}$ & 12.0 & 27.0 \\
$\mathrm{~K}^{-}$ & 1.9 & 5.2 \\
$\overline{\mathrm{P}}$ & 0.0008 & 0.0049
\end{tabular}

